\title{
Simulation of Two-Fluid Flows by the Least-Squares Finite Element Method Using a Continuum Surface Tension Model
}

Jie Wu

Institute for Computational Mechanics in Propulsion

Cleveland, Ohio

Sheng-Tao Yu

NYMA, Inc.

Brook Park, Ohio

Bo-nan Jiang

Institute for Computational Mechanics in Propulsion Cleveland, Ohio

December 1996

Prepared for

Lewis Research Center

Under Cooperative Agreement NCC3-483

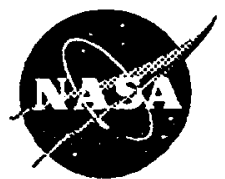

National Aeronautics and

Space Administration

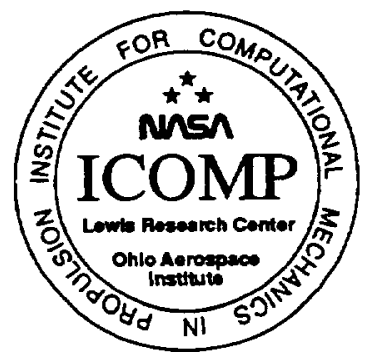





\title{
Simulation of Two-fluid Flows by the Least-Squares Finite Element Method Using a Continuum Surface Tension Model
}

\author{
Jie Wu; Sheng-Tao Yu ${ }^{\dagger}$ and Bo-nan Jiang ${ }^{\ddagger}$ \\ NASA Lewis Research Center, Cleveland, OH 44135
}

\section{ABSTRACT}

In this paper a numerical procedure for simulating two-fluid flows is presented. This procedure is based on the Volume of Fluid (VOF) method proposed by Hirt and Nichols [1] and the continuum surface force (CSF) model developed by Brackbill, et al. [2]. In the VOF method fluids of different properties are identified through the use of a continuous field variable (color function). The color function assigns a unique constant (color) to each fluid. The interfaces between different fluids are distinct due to sharp gradients of the color function. The evolution of the interfaces is captured by solving the convective equation of the color function. The CSF model is used as a means to treat surface tension effect at the interfaces. Here a modified version of the CSF model, proposed by Jacqmin [3], is used to calculate the tension force. In the modified version, the force term is obtained by calculating the divergence of a stress tensor defined by the gradient of the color function. In its analytical form, this stress formulation is equivalent to the original CSF model [2]. Numerically, however, the use of the stress formulation has some advantages over the original CSF model, as it bypasses the difficulty in approximating the curvatures of the interfaces.

The least-squares finite element method (LSFEM) [4] is used to discretize the governing equation systems. The LSFEM has proven to be effective in solving incompressible Navier-Stokes equations and pure convection equations, making it an ideal

*Institute for Computational Mechanics in Propulsion, Ohio Aerospace Institute

†NYMA Technology Inc.

‡Institute for Computational Mechanics in Propulsion, Ohio Aerospace Institute 
candidate for the present applications. The LSFEM handles all the equations in a unified manner without any additional special treatment such as upwinding or artificial dissipation.

Various bench mark tests have been carried out for both two dimensional planar and axisymmetric flows, including a dam breaking, oscillating and stationary bubbles and a conical liquid sheet in a pressure swirl atomizer.

\section{Introduction}

Multi-fluid flows exist in many engineering problems. Examples of such flows include injection molding, metal casting, crystal growth and spray atomization, etc. At the interface of different fluids, surface tension exists as a result of uneven molecular forces. The interface behaves in a way similar to a thinly stretched membrane. The prediction of the evolution of the interface and the treatment of the interface conditions have been a challenging task for numerical simulations.

The pressure jump across the interface is related to the surface tension coefficient $\sigma$ (only constant $\sigma$ is considered), the curvature of the interface $\kappa$, and the viscous stress tensor $\tau^{\mathfrak{v}}$ by the Laplace's formulas [5]:

$$
\left(p_{1}-p_{2}+\sigma \kappa\right) n_{i}=\left(\tau_{1 i k}^{v}-\tau_{2 i k}^{v}\right) n_{k}
$$

where $\mathbf{n}$ is the unit normal of the interface, and the subscripts 1 and 2 denote the two different fluids.

Most existing numerical methods for multiphase/free-surface flows fall into two categories : (1) those which use a fixed grid; and (2) those which allow the grid to deform in time so that it remains surface-intrinsic. In the first category the computational grid is fixed throughout the calculation. An additional variable is used to identify the interface (front). Examples of such methods are the Marker and Cell (MAC) method proposed by Harlow and Welch [6] and the Volume of Fluid (VOF) method by Hirt and Nichols [1]. The MAC method uses massless marker particles which travel with the fluid to trace the fluids and the interface. The VOF method modifies the MAC method by replacing the discrete marker particles with a continuous field variable (color function). This function assigns a unique constant (color) to each fluid and has a sharp gradient at fluid interfaces. Numerical methods in this category are sometimes referred to as "front capturing" methods. Such methods possess great flexibility in handling large deformations and topological changes, as demonstrated by Daly [7] and Harlow and Shannon [8]. The most difficult task with the front capturing approach is to accurately identify the interface and to impose 
the interface condition (1), such as exemplified by the elaborate work of Daly [9]. This difficulty can be alleviated by using a continuum surface force (CSF) model proposed by Brackbill, et inl. [2]. When the CSF model is used, the interface condition (1) is implied in the momentum equations rather than explicitly imposed, thus the location of the interface is no longer explicitly required in the calculation. The computer implementation of the CSF model is therefore relatively simple compared with other approaches. The combination of the VOF method and the CSF model has been used by a number of authors to simulate multi-phase phenomenon involving surface tension and complex topological changes [10,11]. A detailed description of the CSF model will be given in the next section.

For methods in the second category $[12,13,14,15,16]$, which are referred to as "front tracking" methods, imposing the interface condition (1) is easy compared with the first, because the interface always coincides with mesh sides. However, it requires frequent updating of the computational mesh, which can be a complex and time-consuming procedure. In particular, it encounters severe difficulty when the flow experiences severe distortions and complex topological changes.

Another approach which can be regarded as a combination of the above is the fronttracking method introduced by Unverdi and Tryggvason [17]. This approach uses a fixed, structured grid to represent the flow field. A separate, unstructured grid is used to represent the interface. The interface is explicitly tracked and kept at a constant thickness of the order of the mesh size. This ensures that the interface will not be smeared by numerical diffusion. Much success has been achieved in solving a variety of two-fluid flow problems using this approach $[18,19]$. The difficulty with this approach is the handling of complex topological changes.

In this paper numerical solutions to problems involving two immiscible fluids are sought. A large number of such problems deal with the interaction between a liquid and air, which are often simplified as free-surface problems. In the free-surface formulation the flow equations are solved only for the liquid, and zero traction is assumed on the interface. In the present calculation such problems are treated as true two-fluid cases. The VOF approach and a modified version of the CSF model, proposed by Jacqmin [3], are used in the simulation. The LSFEM [4] is used to discretize the governing system of equations. The LSFEM has proven to be effective in solving the Navier-Stokes equations for incompressible flows $[4,20]$ and pure convection equations [21,22], making it an ideal candidate for the present applications. Some preliminary two dimensional calculations for two-fluid problems have been reported in [22]. The contents of this paper are arranged as follows: In the next section the CSF model will be elaborated. In Section 3 the governing equations and the discretization procedure will be presented. Some two dimensional and axisymmetric numerical tests will be presented in Section 4 . The first test case is a two dimensional dam breaking problem. The numerical results by the 
present approach are compared with experimental data and with results obtained by other approaches. Good agreement is observed. The second test case deals with an oscillating bubble for both two dimensional and axisymmetric cases. The third case is a two-liquid interface problem in a closed box. The last test case simulates the formation of a liquid sheet in a pressure swirl atomizer (a Simplex nozzle). Finally, some conclusions are given in Section 5 .

\section{The continuum surface force model}

The continuum surface force (CSF) model was introduced by Brackbill, et al. [2] as a means of treating the surface tension effect in the VOF method. By using this model, the difficulty in imposing the interface constraints (1) was alleviated. The basic idea of the CSF model is to regard the interface between two fluids as a transition region with a finite thickness, instead of a zero-thickness membrane. The surface tension effect is interpreted as a continuous body force spread across the transition region, which acts as a source term in the momentum equations. $\mathrm{By}$ using the CSF model, the interface condition (1) no longer needs to be explicitly imposed, as it is already implied in the momentum equations. The body force in the CSF model is obtained through some differential operations on the spatially continuous color function, as follows:

$$
\mathbf{f}=\frac{\sigma}{[C]} \kappa \nabla C
$$

where $\mathbf{f}$ is the body force, $\sigma$ and $\kappa$ are the surface tension coefficient and the curvature of the interfaces, the same as in (1),C is the color function, and $[C]$ denotes the jump of $C$ across the interface. The curvature $\kappa$ in the above formulation is calculated from:

$$
\kappa=-(\nabla \cdot \mathbf{n})
$$

where $\mathbf{n}$ is the unit normal to the surface, which is obtained from:

$$
\mathbf{n}=\frac{\nabla C}{|\nabla C|}
$$

Jacqmin [3] derived the CSF model through the analysis of tension energy. He pointed out that the surface tension force can be expressed as the divergence of a stress tensor which is uniquely defined by the gradient of the color function. Let $\tau_{i j}$ denote the the stress components in a Cartesian coordinate system. They are related to the color function $C$ as :

$$
\tau_{i j}=-\frac{\sigma}{[C]} \frac{1}{|\nabla C|}\left(\frac{\partial C}{\partial x_{i}} \frac{\partial C}{\partial x_{j}}-\delta_{i j} \frac{\partial C}{\partial x_{k}} \frac{\partial C}{\partial x_{k}}\right)
$$




$$
=-\frac{\sigma}{[C]}\left(\frac{1}{|\nabla C|} \frac{\partial C}{\partial x_{i}} \frac{\partial C}{\partial x_{j}}-\delta_{i j}|\nabla C|\right)
$$

where $\delta_{i j}$ is the Kronecker delta.

The volumetric body force caused by the surface tension is expressed as:

$$
f_{i}=\frac{\partial \tau_{i j}}{\partial x_{j}}
$$

It can be easily verified that $\mathbf{f}$ defined by Eqs. (5) and (6) is analytically equivalent to that of the original CSF model given by Eqs. (2)-(4). The advantage of using the formulation given by Eqs. (5) and (6) instead of the original CSF model is that Eqs. (5) and (6) do not require the explicit calculation of the normalized gradient term in the right hand side of Eq. (4), whose definition is not clear when the denominator $|\nabla C|$ approaches zero. In Eqs. (5) and (6) both $\tau$ and $f$ are well defined in the whole domain; and naturally vanish when $|\nabla C|$ becomes zero. An additional advantage of using the stress tensor $\tau$ is that it can be regarded as part of the momentum flux. In many numerical procedures $\tau$ can be used directly and there is no need to calculate $\mathbf{f}$.

It is worth pointing out that the stress tensor $\tau$ also describes more directly the fluid physics at the interface than the force term $\mathbf{f}$. To illustrate this argument, let 1 and 2 denote, respectively, the directions along and normal to the interface in two dimensions (Figure 1). We have:

$$
(\nabla C)_{1}=0, \quad(\nabla C)_{2}=|\nabla C|
$$

The stress components are (for clarity the definition of stress is also given in Figure 1):

$$
\tau_{11}=\frac{\sigma}{[C]}|\nabla C|, \quad \tau_{22}=\tau_{12}=\tau_{21}=0
$$

From the above we have:

$$
\int_{s_{2}} \tau_{11} d s=\sigma
$$

This indicates that when the interface thickness approaches zero, the stress tensor defined by Eq. (5) also approaches the true surface tension. The fact that $\tau_{11}$ is always positive in the transition region reflects that the interface is like a stretched membrane. $\tau$ is also useful in situations when the surface tension coefficient $\sigma$ is no longer constant. 

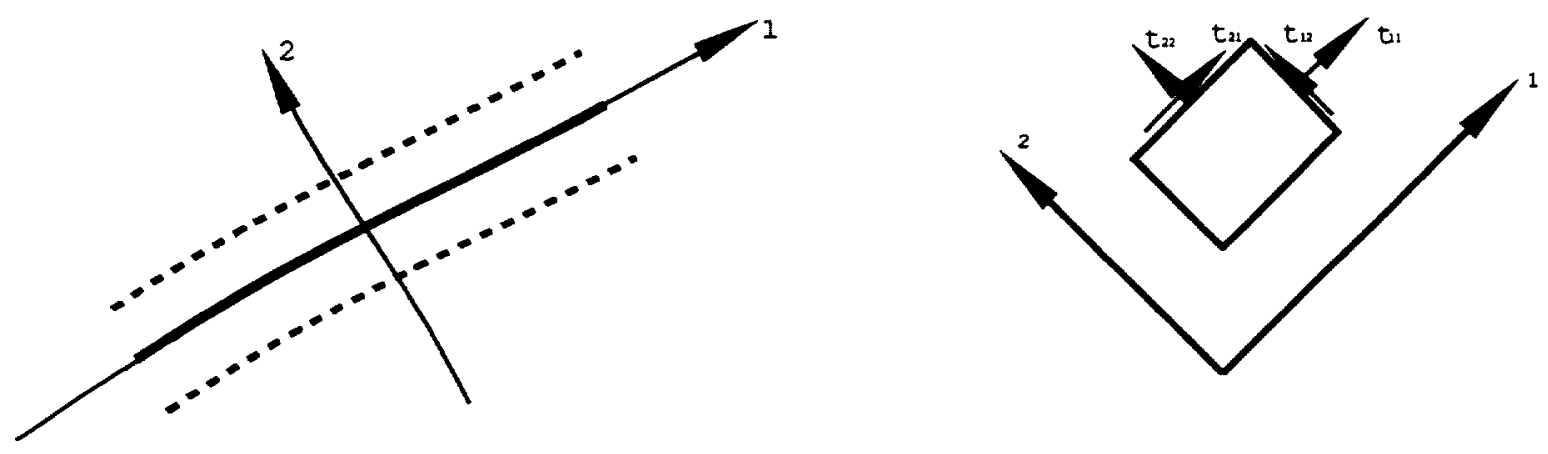

Figure 1: The continuum surface and the stress components

It should be pointed out that Lafaurie, et al. [11] introduced a capillary pressure tensor $\mathbf{T}$, and used it as a correction to the momentum stress tensor. $\mathbf{T}$ is defined as:

$$
\mathbf{T}=\sigma(\mathbf{I}-\mathbf{n} \otimes \mathbf{n})|\nabla C|
$$

where $n$ is the unit normal to the surface as defined by Eq. (4). It is easy to verified that $\tau$ in Eqs. (5) and (6) and $\mathbf{T}$ in Eq. (7) are also analytically equivalent except for a minus sign. Again the advantage of using $\tau$ is that it bypasses the calculation of $\mathbf{n}$.

\section{The Governing Equations and the Discretiza- tion Procedure}

The governing equations for the two-fluid problems are the standard Navier-Stokes equations. Incompressibility is assumed for both fluids. In order to use the $C_{0}$ element in the LSFEM formulation, the governing equations need to be re-written as a first-order system. Here we choose the following velocity-pressure-vorticity form:

$$
\begin{aligned}
& \nabla \cdot \mathbf{u}=\mathbf{0} \\
& \rho \frac{\partial \mathbf{u}}{\partial t}+\rho(\mathbf{u} \cdot \nabla) \mathbf{u}+\nabla p+\mu(\nabla \times \boldsymbol{\omega}) \\
& -\left(\nabla \mu \cdot \nabla \mathbf{u}+\nabla \mu \cdot(\nabla \mathbf{u})^{T}\right)=\mathbf{f}
\end{aligned}
$$

in which $\rho$ is the density, $\mathbf{u}$ is the velocity vector, $p$ is the pressure, $\omega$ is the vorticity vector, $\mu$ is the dynamic viscosity, and $\mathbf{f}$ is the body force, which generally consists 
of the surface tension effect given by Eq. (3) and the gravitational force. The terms in the last pair of brackets on the left hand side represents the effect of non-uniform viscosity.

The vorticity $\boldsymbol{\omega}$ is defined by the velocity $\mathbf{u}$ as:

$$
\boldsymbol{\omega}=\nabla \times \mathbf{u}
$$

From the above we immediately have:

$$
\nabla \cdot \boldsymbol{\omega}=0
$$

The above equation system, Eqs. (8)-(11), has been used by Jiang et al. [23] in the theoretical analysis of Navier-Stokes equations systems; and has been extensively used as the basis of LSFEM calculations [4, 20, 24].

The fluids are identified by the different value of the color function $C$, which is convected by the flow field:

$$
\frac{\partial C}{\partial t}+(\mathbf{u} \cdot \nabla) C=0
$$

Some numerical examples in this paper deal with axisymmetric (non-swirling and swirling) flows, which require the above equations to be recast into a cylindrical coordinate system. These equations will be given in Appendix A.

Fluid properties such as the density and the viscosity are assumed to be distributed in the same manner as $C$, i.e.,

$$
\begin{aligned}
\rho & =\rho_{1}+\frac{\rho_{2}-\rho_{1}}{C_{2}-C_{1}}\left(C-C_{1}\right) \\
\mu & =\mu_{1}+\frac{\mu_{2}-\mu_{1}}{C_{2}-C_{1}}\left(C-C_{1}\right)
\end{aligned}
$$

The governing equations, Eqs. (8)-(12), are first discretized in time. The backward Euler difference scheme is used:

$$
\begin{aligned}
& \nabla \cdot \mathbf{u}^{n+1}=0 \\
& \rho^{n+1} \frac{\mathbf{u}^{n+1}-\mathbf{u}^{n}}{\Delta t}+\rho^{n+1}\left(\mathbf{u}^{n+1} \cdot \nabla\right) \mathbf{u}^{n+1}+\nabla p^{n+1}+\mu^{n+1}\left(\nabla \times \omega^{n+1}\right) \\
& -\left(\nabla \mu^{n+1} \cdot \nabla \mathbf{u}^{n+1}+\nabla \mu^{n+1} \cdot\left(\nabla \mathbf{u}^{n+1}\right)^{T}\right)=\mathrm{f}^{n+1} \\
& \boldsymbol{\omega}^{n+1}=\nabla \times \mathbf{u}^{n+1} \\
& \frac{C^{n+1}-C^{n}}{\Delta t}+\left(\mathbf{u}^{n+1} \cdot \nabla\right) C^{n+1}=0
\end{aligned}
$$


The superscript ' $n$ ' denotes the previous time step and ' $n+1$ ' denotes the current time step.

The above equations, Eqs. (15)-(18), are further linearized. To ensure time-accuracy for time-dependent problems linearization iterations are performed within each time step (typically three iterations are used). For steady state problems only one linearization iteration is performed in each time step. Let $r$ denote the number of linerization iterations, the linearization is carried out as follows:

$$
\begin{aligned}
& \nabla \cdot\left(\mathbf{u}^{n+1}\right)_{[r]}=0 \\
& \left(\rho^{n+1}\right)_{[r-1]} \frac{\left(\mathbf{u}^{n+1}\right)_{[r]}}{\Delta t}+\left(\rho^{n+1}\right)_{[r-1]}\left(\left(\mathbf{u}^{n+1}\right)_{[r-1]} \cdot \nabla\right)\left(\mathbf{u}^{n+1}\right)_{[r]} \\
& +\nabla\left(p^{n+1}\right)_{[r]}+\left(\mu^{n+1}\right)_{[r-1]} \nabla \times\left(\boldsymbol{\omega}^{n+1}\right)_{[r]} \\
& =\left(\mathbf{f}^{n+1}\right)_{[r-1]}+\left(\rho^{n+1}\right)_{[r-1]} \frac{\mathbf{u}^{n}}{\Delta t} \\
& +\left(\nabla\left(\mu^{n+1}\right)_{[r-1]} \cdot \nabla\left(\mathbf{u}^{n+1}\right)_{[r-1]}+\nabla\left(\mu^{n+1}\right)_{[r-1]} \cdot\left(\nabla\left(\mathbf{u}^{n+1}\right)_{[r-1]}\right)^{T}\right) \\
& \left(\boldsymbol{\omega}^{n+1}\right)_{[r]}-\nabla \times\left(\mathbf{u}^{n+1}\right)_{[r]}=0 \\
& \frac{\left(C^{n+1}\right)_{[r]}}{\Delta t}+\left(\left(\mathbf{u}^{n+1}\right)_{[r]} \cdot \nabla\right)\left(C^{n+1}\right)_{[r]}=\frac{C_{[r-1]}^{n}}{\Delta t}
\end{aligned}
$$

In the above $\left(C^{n+1}\right)_{[0]}=C^{n},\left(\mu^{n+1}\right)_{[0]}=\mu^{n}$ and $\left(\mathbf{u}^{n+1}\right)_{[0]}=\mathbf{u}^{n}$.

At this stage the standard LSFEM procedure [4] is introduced for spatial discretization. We note that the solution of the flow field $\left(\left(\mathbf{u}^{n+1}\right)_{[r]},\left(p^{n+1}\right)_{[r]},\left(\boldsymbol{\omega}^{n+1}\right)_{[r]}\right)$ does not require the knowledge of $\left(C^{n+1}\right)_{[\tau]}$, Thus for each linearization iteration, Eqs. $(19-21)$ are first solved. Then the newly obtained $\left(\mathbf{u}^{n+1}\right)_{[r]}$ is used in Eq. (22) to solve for $\left(C^{n+1}\right)_{[r]}$. We use reduced integration (one point Gauss quadrature for bi-linear elements) in the solution of Eqs. (19)-(21). The reason for so doing is that, as pointed out by Jiang and Povinelli [4], the LSFEM with Gaussian quadrature is equivalent to the collocation least-squares method; and the use of reduced integration makes the total number of collocation points compatible with the total number of unknowns and has proved to result in better accuracy [21]. For Eq. (22), however, we have used exact numerical integration $(2 \times 2$ Gauss points for bi-linear elements), because using reduced integration sometimes produces oscillatory solutions for the color function. Such oscillations will further result in unacceptable errors in the tension force, which is obtained by twice differentiating the color functions. In order to avoid excessive numerical diffusion and to maintain time accuracy, the time step is chosen so that the corresponding CFL number is less than or close to unity for true transient problems. If only steady state results are of interest, much larger time steps are used. A Jacobi-preconditioned conjugate gradient (JCG) method has 
been used to solve the algebraic equations. Since the LSFEM always results in a symmetric, positive-definite matrix, the JCG method is guaranteed to converge to the correct solution. Since the JCG iterations can be carried out in an elementby-element fashion such that no global matrix needs to be stored [25], very large problems can be solved with moderate computer memory requirements [20].

The body force term $\left(\mathbf{f}^{n+1}\right)_{[r-1]}$ in Eq. (20) is calculated using Eqs. (5) and (6). The finite element solution of $\tau$ obtained from Eq. (5) is discontinuous across element boundaries. In order to use Eq. (6) to obtain the body force $f$, the $\tau$ field needs to be spatially continuous. Such a continuous field can be obtained by treating the discontinuous field with various recovery procedures (see [26] and the references therein). In this paper only bi-linear finite element is used for the discretization and a simple averaging method [27] is used for the recovery procedure. For higher order elements, the superconvergent patch recovery (SPR) [26] should be used. The recovery procedure is also used to obtain a continuous $f$ field.

\section{Numerical Examples}

\subsection{A Broken Dam Problem}

This problem has been used by many as a test case for simulating free-surface problems. Experimental data for this case are available [28]. Here the problem is solved as a two-fluid problem involving both the water and air. Zero surface tension and slippery walls (left and bottom sides) are assumed. On the top and right sides zero pressure is imposed. In addition, if inflow is detected on the top and right sides, the density of air is imposed. The computational domain is 2 units high and 6 units long. Initially, water occupies a $1 \times 1$ area at the bottom left corner. The computational mesh consists of $120 \times 40$ uniform bilinear quadrilateral elements. The time step is $\Delta t=0.05$. The nondimensionalized gravitational acceleration, $g$, is taken to be unity. The viscosity is set at $3.05 \times 10^{-5}$ for water (which is the same choice as in [29]) and $3.05 \times 10^{-8}$ for air. The densities for water and air are 1 and 0.001 , respectively.

Figure 2 shows free surface profiles (a contour line of density at $\rho=0.5$ ) and the pressure contours at various times. The calculated water front location and water column height are compared with the experimental data in Figure 3. It can be seen that the calculated results are in good agreement with the experimental data [28] and with the calculation results in [29]. 

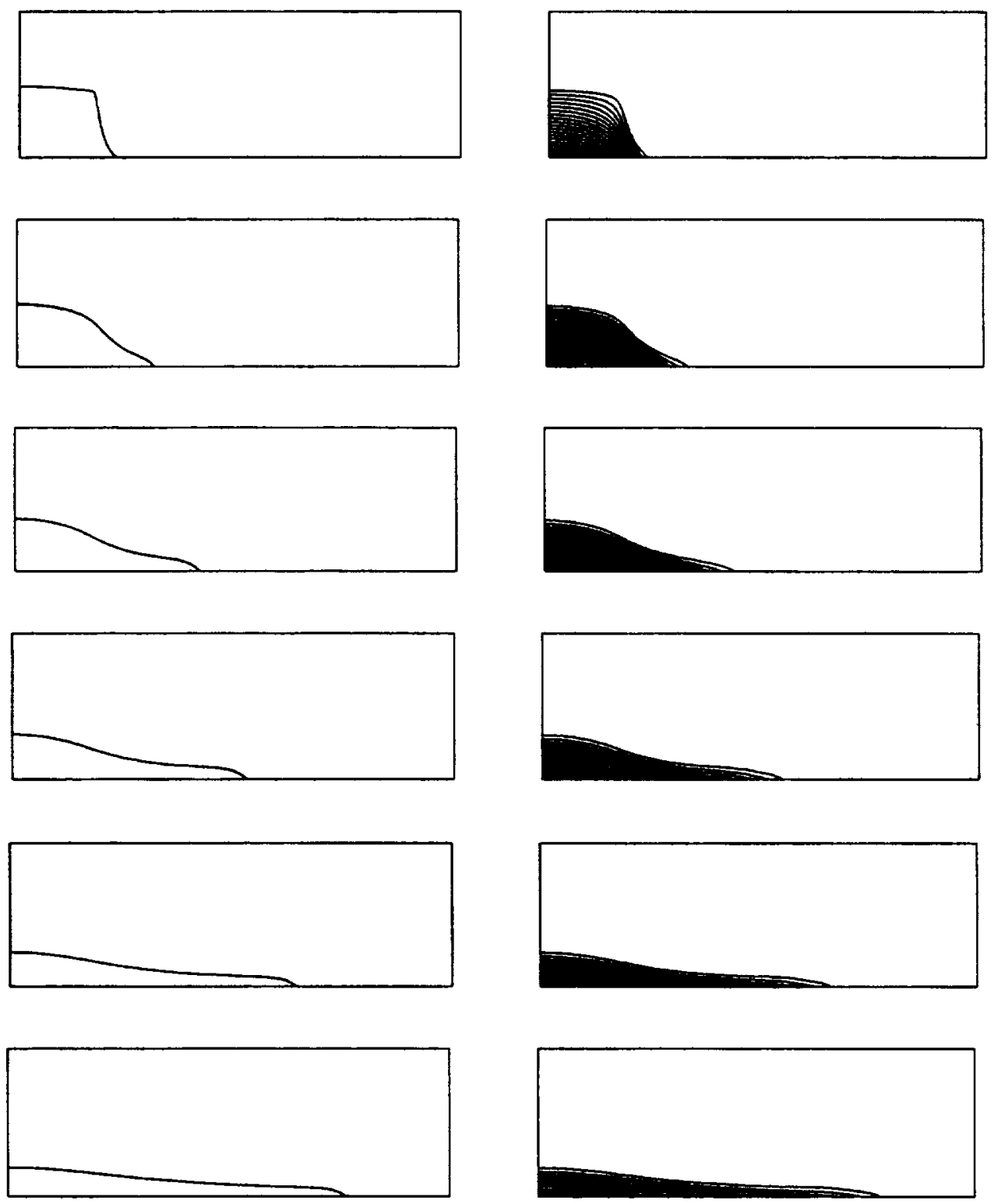

Figure 2: A broken dam, free surface profile and pressure contours. From top to bottom: $t=0.5,1.0,1.5,2.0,2.5,3.0$. 

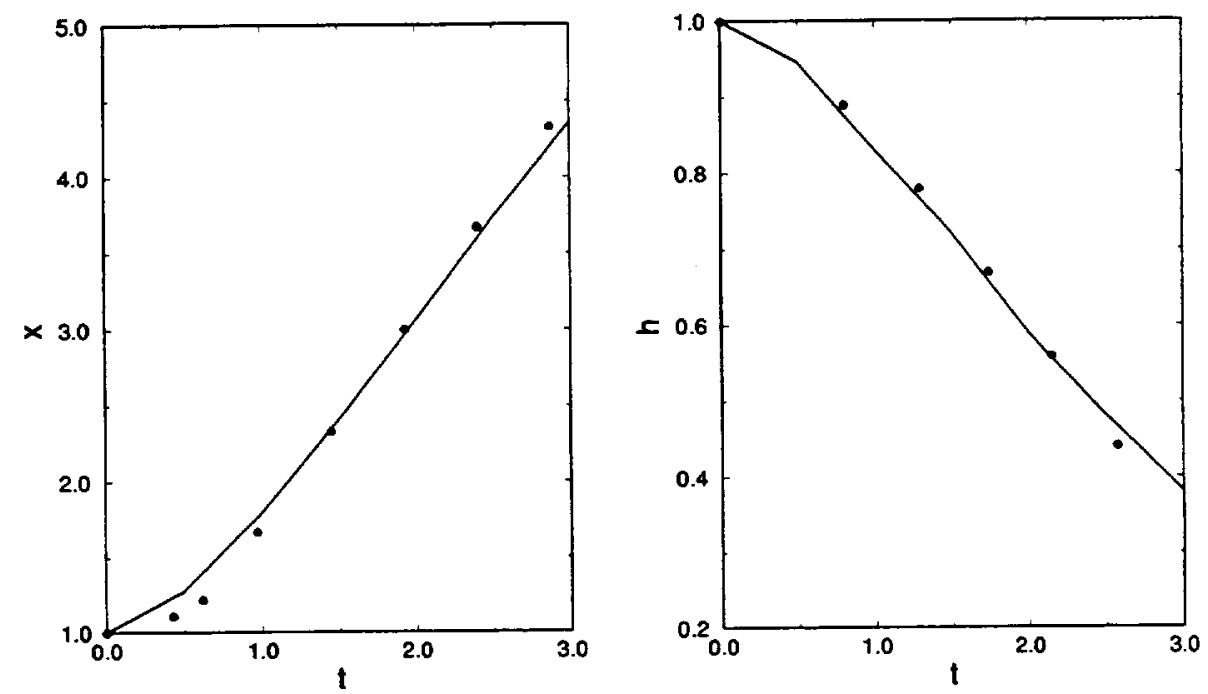

Figure 3: A broken dam, front location and water column height. solid line calculated results; dots - experimental data.

\subsection{An Oscillating Bubble}

In this example the oscillation of a bubble due to surface tension is studied. Both two-dimensional planar and axisymmetric cases are considered. The two dimensional case was simulated by Fyfe et al. [13] using a Lagrangian approach. The computational domain in this calculation is $4 \times 12$ units with the symmetry plane/axis along the longer side. The computational mesh consists of $40 \times 120$ uniform bilinear quadrilateral elements. Initially the shape of the bubble is elliptical, given by $x^{2} / 4+z^{2}=1$. The density of the fluid inside and outside the bubble is 1.5 and 0.5 , respectively. The dynamic viscosity is 0.01 for both fluids. A time step of $\Delta t=0.05$ is used in both cases. After the calculation reaches $t=15.0$, viscosity is increased to 1.0 to allow the solution to converge to steady state. When steady state is reached the sizes of the bubbles are 1.40 and 1.58 , respectively, while the theoretical values are 1.41 and 1.59. At steady state, it is verified that the Laplace's formulas Eq. (1) for the pressure jump is satisfied.

In Figs. 4 and 5 the interface shapes at different times are shown for two dimensional and axisymmetric cases, respectively. The corresponding time history of the interface locations on the $x$-axis is shown in Figure 6. The oscillation periods are estimated (based on the first two cycles) to be 7.6 for the two-dimensional case and 7.2 for the axisymmetric case. Theoretical solutions of the oscillation periods are available from a linear analysis for small amplitudes (see [13, 19] for details and references), which for this example are 6.1 for the two-dimensional case and 6.3 for the axisymmetry case. Since in the calculations both nonlinear and viscous effects 

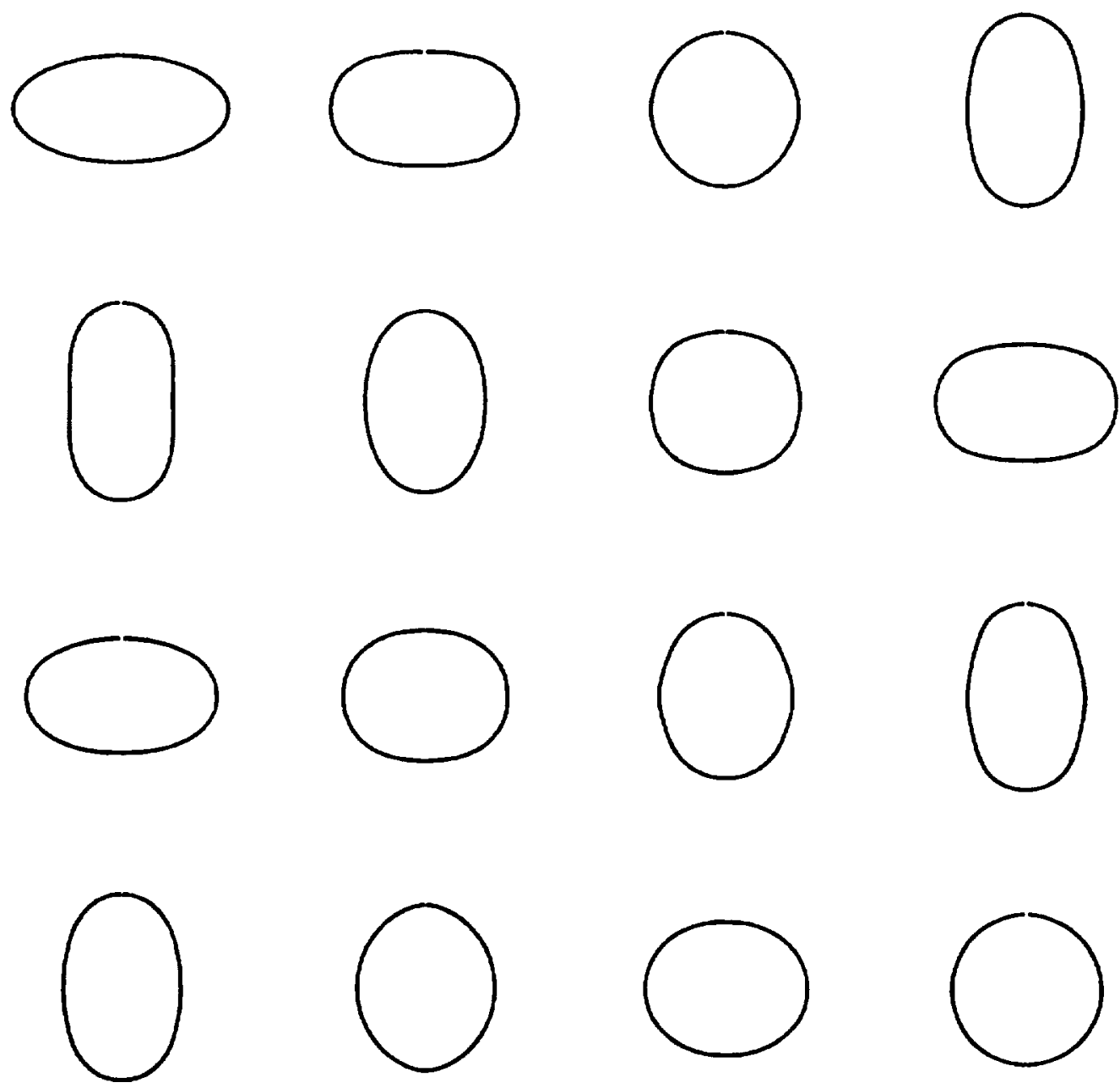

Figure 4: An oscillating bubble, interface profile for two dimensional case, $t=$ $0,1,2,3,4,5,6,7,8,9,10,11,12,13,14,40$, respectively 

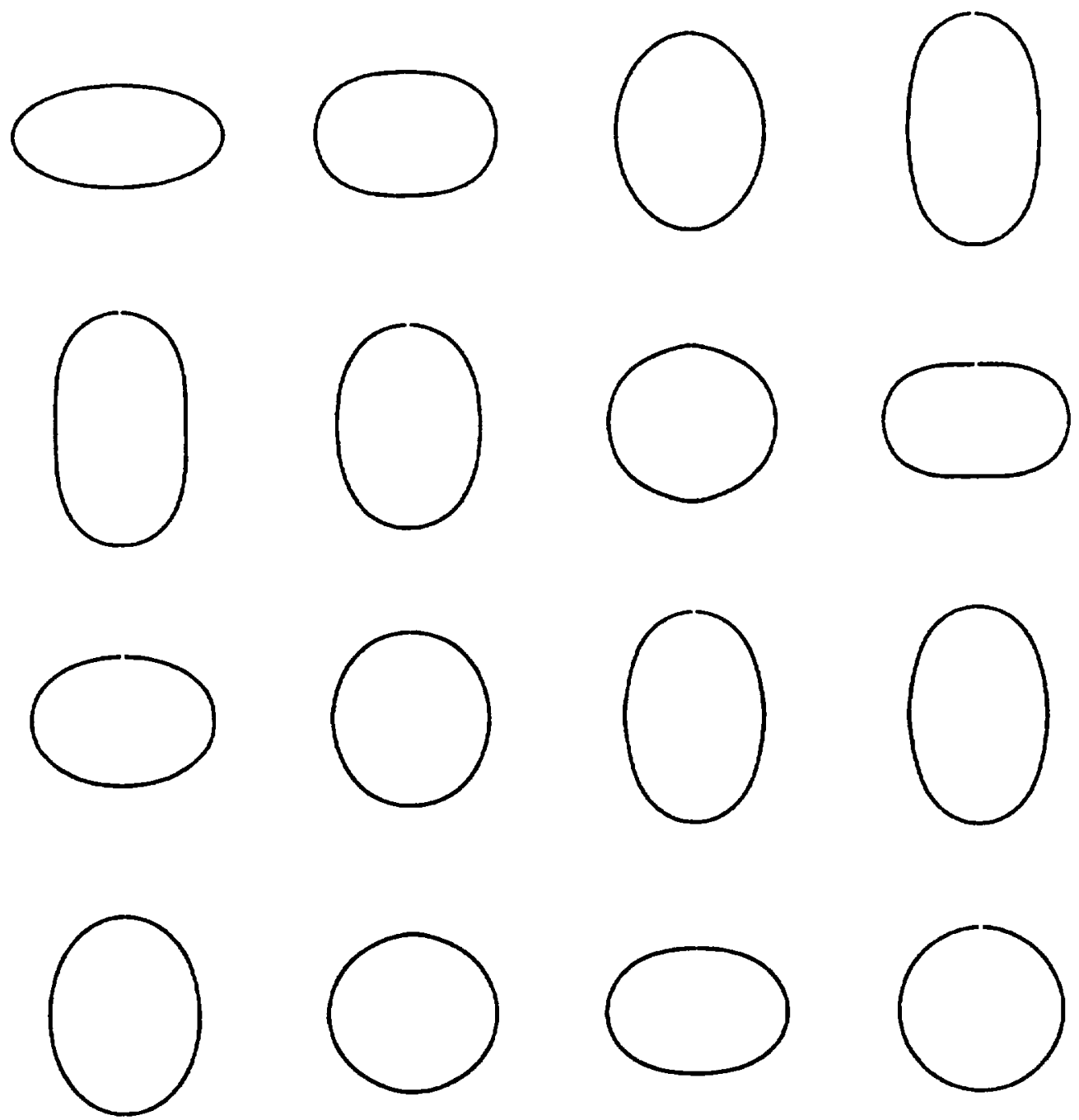

Figure 5: An oscillating bubble, interface profile for axisymmetric case, $t=$ $0,1,2,3,4,5,6,7,8,9,10,11,12,13,14,40$, respectively 

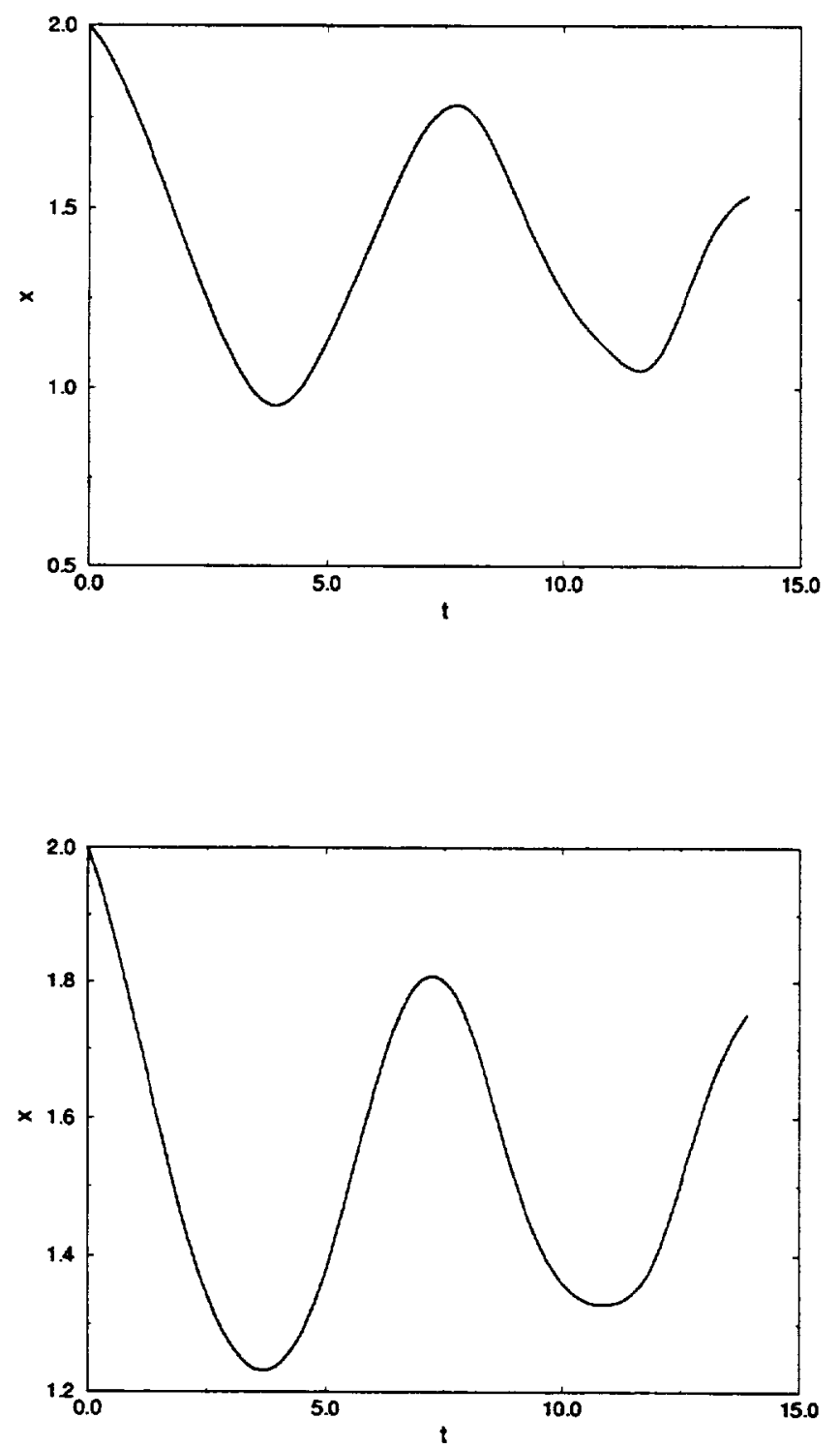

Figure 6: An oscillating bubble, time history of the interface location on the $x$-axis. top: two dimensional case; bottom: axisymmetric case. 
are present, the theoretical solutions can be used only as references. The current numerical simulation predicts higher values of the oscillation periods than linear theory, which is consistent with the experience of Fyfe et al. [13].

\subsection{Two-liquid Interface Problem}

This example is due to Tezduyar, et al. [16]. In this problem, a two dimensional $0.8 \times 0.6$ box is fully occupied by two liquids of equal volume. The density of the fluids are 1.0 and 2.0, respectively. The gravitational acceleration is 0.294 . The lighter liquid is placed on top of the heavier one. Initially the interface is a straight line, with a slope of 0.25 . The dynamical viscosity is 0.001 (the same for both liquids). Zero surface tension is assumed. The computational mesh consists of $21 \times 42$ uniform bilinear elements. A time step of $\Delta t=0.25$ is used throughout the calculation (which corresponds to a CFL number close to 1 when the velocity reaches its maximum at approximately $t=6$ ). Slippery wall conditions are used on all four sides. Figure 7 shows the interface profile and velocity field at different times. Figure 8 shows the time history of interface location on the left and right hand side walls (relative to and normalized by the average height). The results agree well with those presented in [16].

\subsection{Flow in a Pressure Swirl Atomizer}

This example deals with the swirling flow in a pressure swirl atomizer (a Simplex nozzle). Simplex nozzles are widely used as fuel atomizers in gas turbine engines. The formation of a conical liquid sheet is characteristic of such nozzles. Prediction of the thickness of the liquid sheet and the exit angle for various inlet conditions provides helpful information in the design process. The computational domain for the current simulation is shown in Figure 9. The liquid enters to the swirl chamber though a number of inlet slots. For the present calculation, axisymmetry is assumed. Gravitational effects are ignored. The computational mesh, shown in Figure 10, consists of 3600 bilinear elements with 3741 nodes. Initially the whole domain is filled with motionless air. At $t>0$ liquid begins to stream into the domain through the inlet slots. At the inlet, values of the liquid density and velocity are prescribed. The prescribed values are: $u_{r}=-4.8, u_{\theta}=8.0, u_{z}=3.6, \rho=0.1$ The density of the air is $\rho=0.01$. The dynamic viscosity for both fluids are $\mu=0.005$. For nodes on the free boundary, the density of air is imposed if inflow is detected, nothing is imposed if outflow is detected. Slippery boundary conditions are imposed on portions of the wall; and non-slip conditions are imposed elsewhere on the wall, as shown in Figure 9. The pressure is specified on the top left corner of the domain. A 

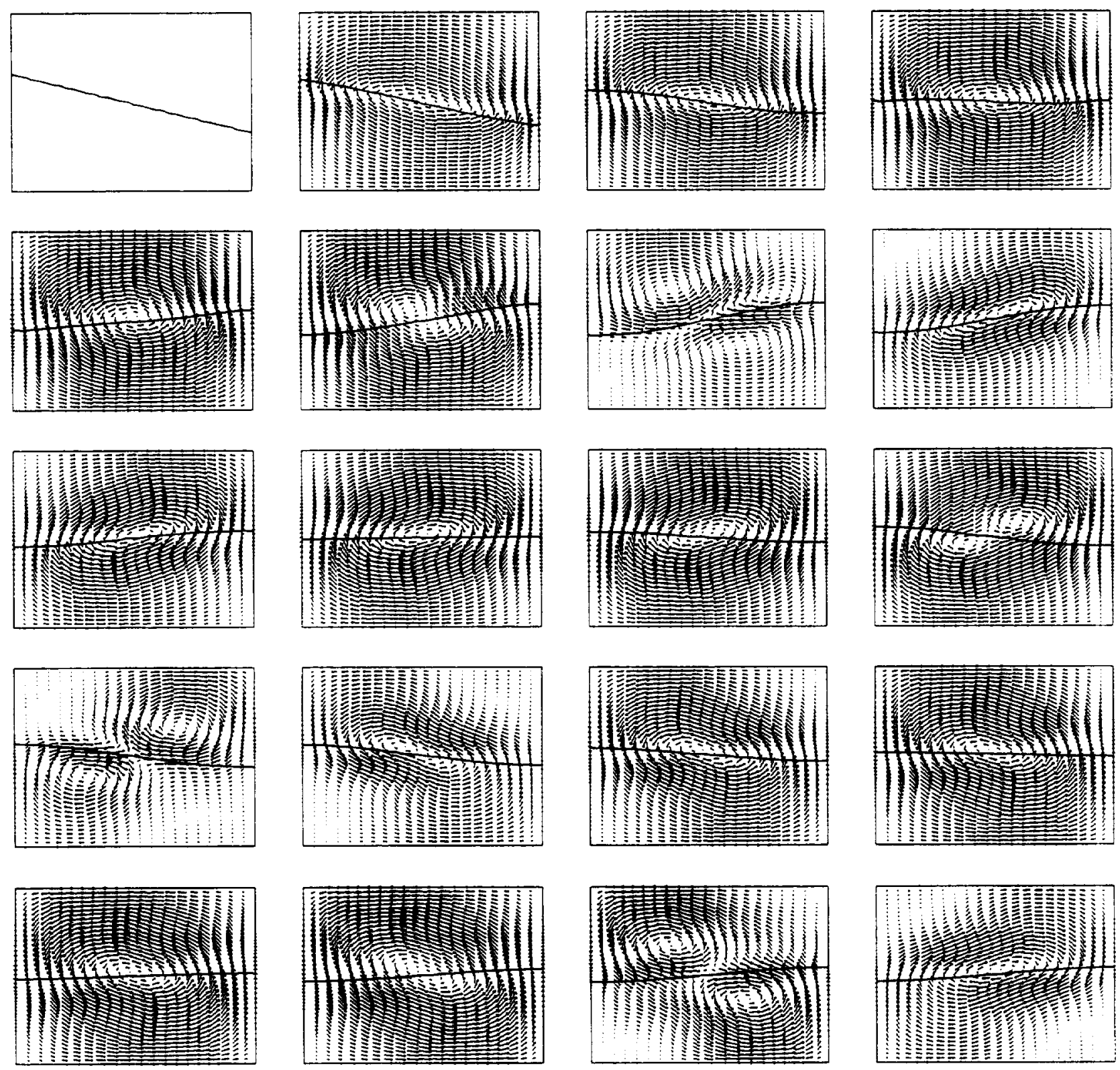

Figure 7: Two-liquid interface problem, interface profile and velocity field, $t=$ $0,1,2,3,4,5,6,7,8,9,10,11,12,13,14,15,16,17,18,19$, respectively 


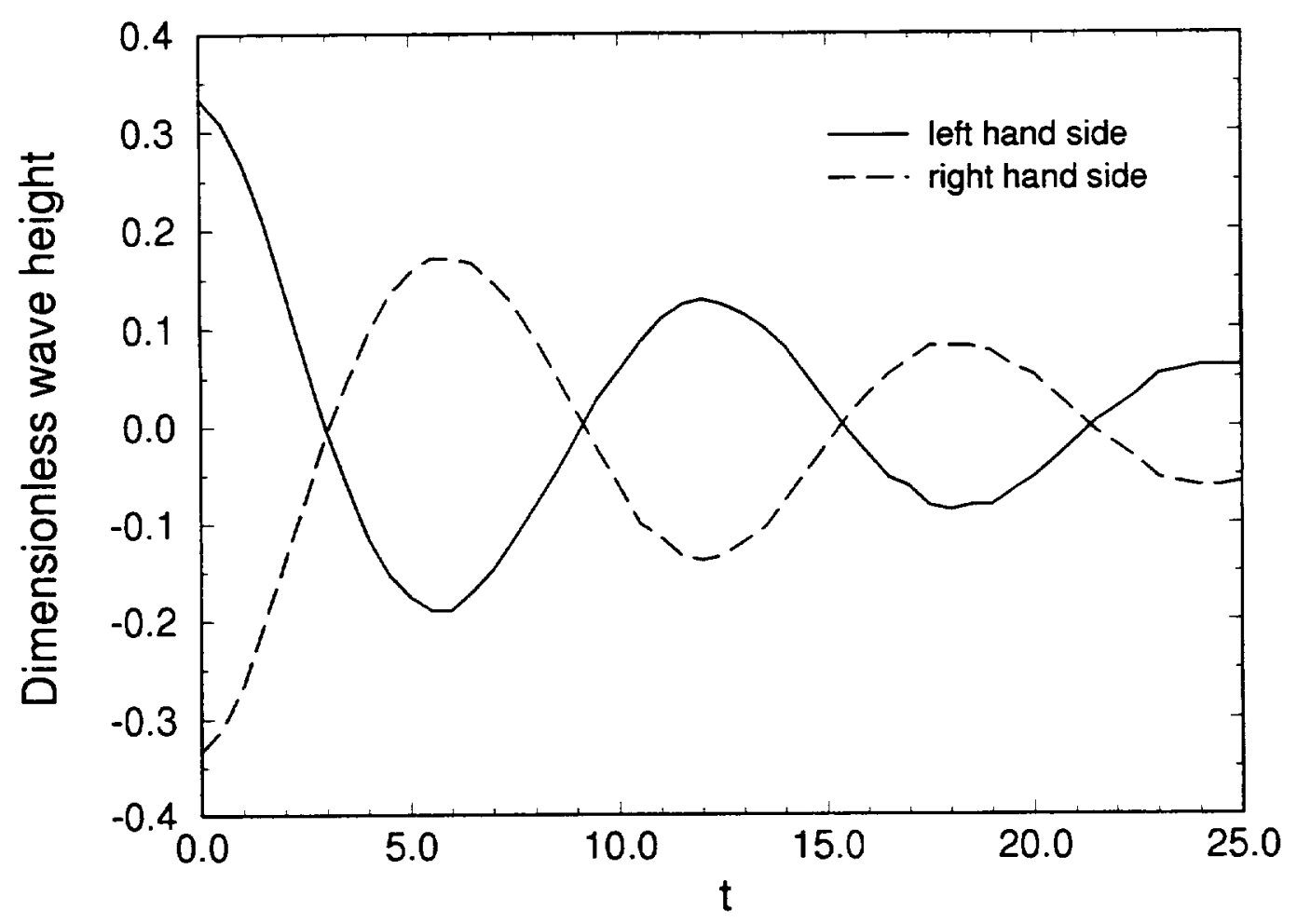

Figure 8: Two-liquid interface problem, time history of interface location on the left and right hand side walls 
constant time step $\Delta t=0.1$ is used throughout the calculation. As only the steady state solution is of interest, a linearization iteration is performed only once in each time step. Figure 10 shows the steady state interface profile. The formation of the conical liquid sheet is successfully predicted in this simulation. This is a preliminary simulation and no attempt has been made to compare computational results with measured data. At the moment, no experimental data are available for comparison.

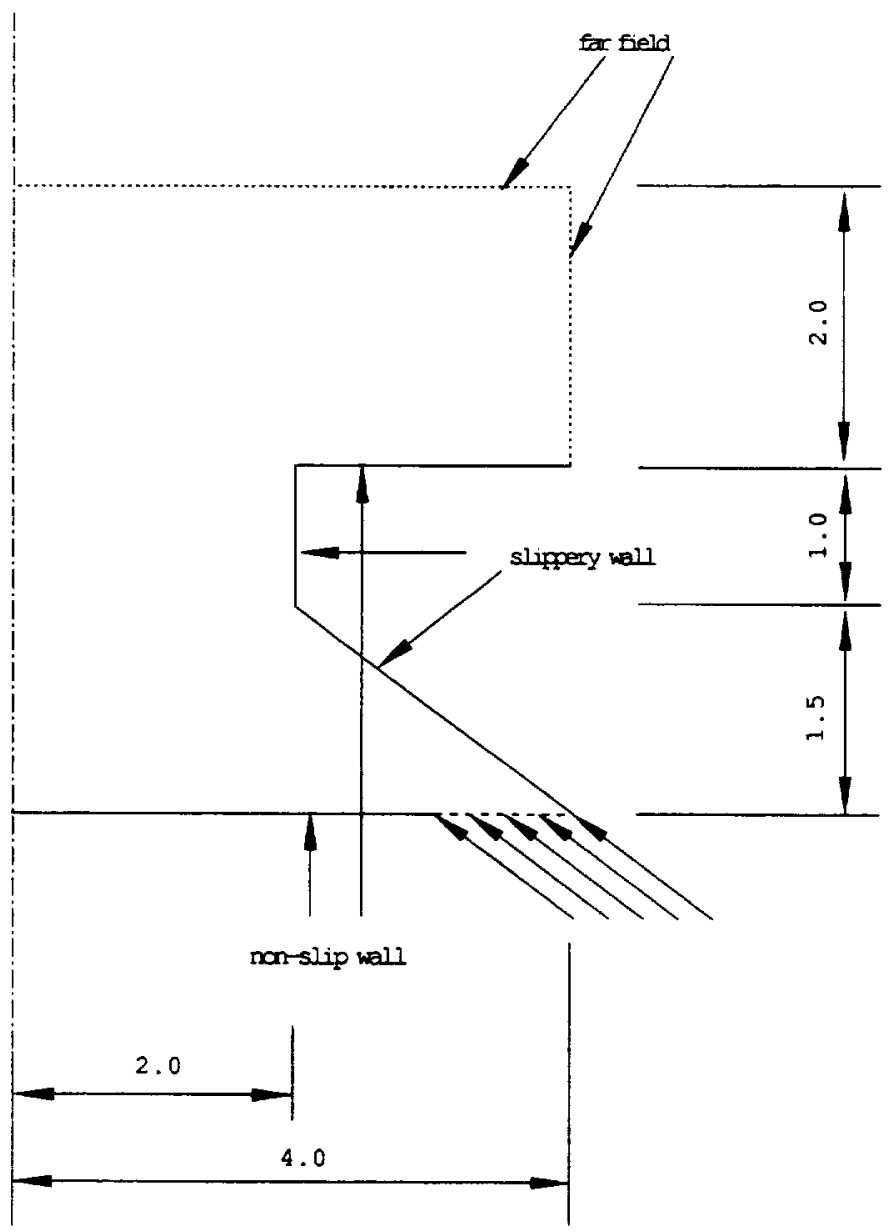

Figure 9: Flow in a pressure swirl atomizer, the problem definition 

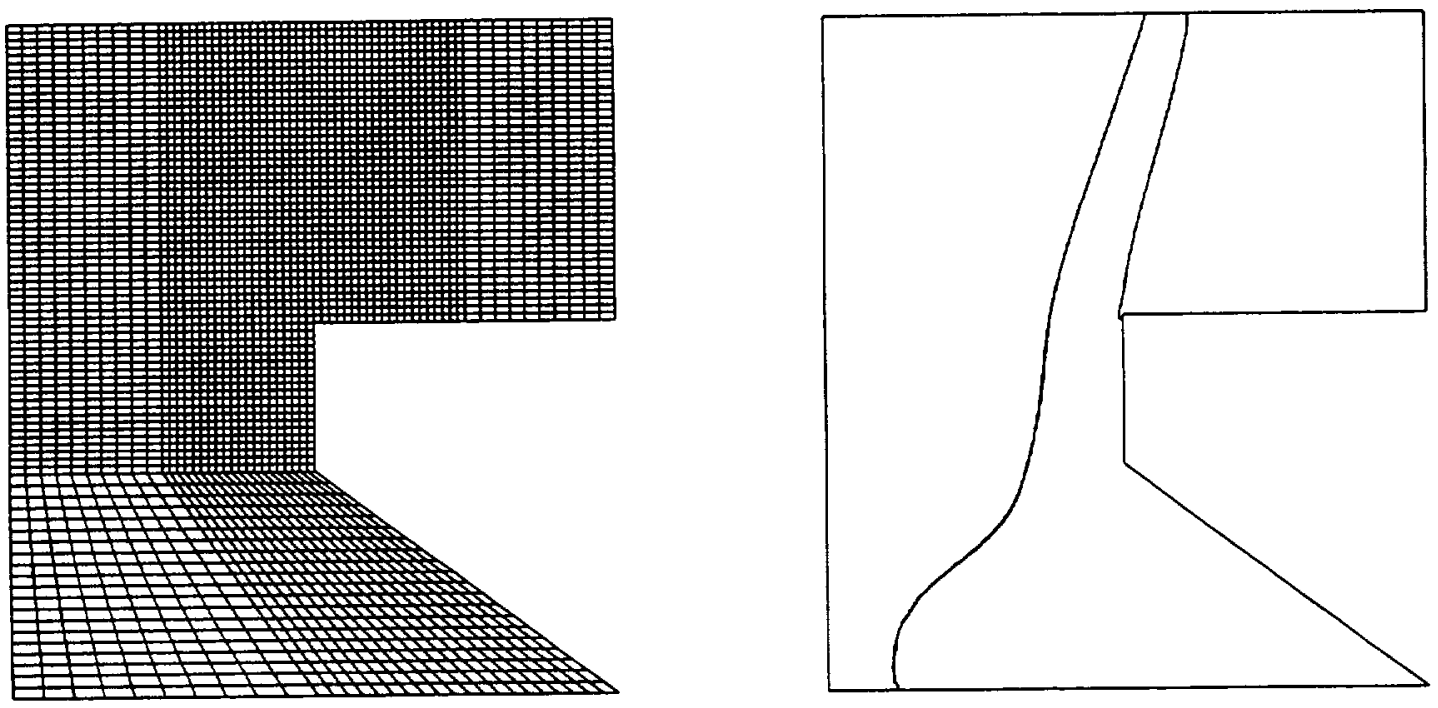

Figure 10: Flow in a pressure swirl atomizer, the computational mesh and the interface profile

\section{Concluding Remarks}

In this paper a numerical procedure based on a CSF model and the LSFEM is presented for two-fluid flow problems. Numerical tests carried out on a number of two dimensional planar and axisymmetric flows indicate that this approach is capable of simulating such flow phenomena. The present approach has the advantage in the ability to handle complex topological changes such as the breakup of a liquid jet. Another advantage is the ability to handle complex geometrical configurations in practical engineering environments, since the LSFEM is based on totally unstructured grids. Currently the interface is typically spread over $3-5$ grids. A change of the interface thickness is also observed in the calculations. The analysis by $\mathrm{Haj}$ Hariri [30] indicates that the migration velocity is strongly affected by the smearing of interface. Thus, to ensure good accuracy of the simulation a fine grid would have to be used. Improvement of the accuracy of the numerical scheme is expected by introducing higher order discretization schemes in both time and space. Our tests [22] indicate that the discontinuity can be modeled within 3 grids, even when the interface undergoes very large deformation due to a highly vortical flow field. 


\section{References}

[1] C. W. Hirt and B. D. Nichols, "Volume of Fluid (VOF) Method for the Dynamics of Free Boundaries", J. Comp. Phys. , 39 , 201-225 (1981).

[2] J. U. Brackbill, D. B. Kothe and C. Zemach, "A Continuum Method for Modeling Surface Tension", J. Comp. Phys., 100, 335-354 (1992).

[3] D. Jacqmin, "Three-Dimensional Computations of Droplet Collisions, Coalescence, and Droplet/Wall Interactions Using a Continuum Surface-Tension Method", AIAA-95-0883.

[4] B. N. Jiang and L. A. Povinelli, "Least-squares finite element method for fluid dynamics", Comp. Meth. Appl. Mech. Engng., 81, 13-37 (1990).

[5] L. D. Landau and E. M. Lifshitz, Fluid Mechanics, Pergammon, New York, 1959.

[6] F. H. Harlow and J. E. Welch, "Numerical Calculation of Time-Dependent Viscous Incompressible Flow of Fluid with Free-Surface", Phys. Fluids, 8, 21822189 (1965).

[7] B. J. Daly, "Numerical Study of Two Fluid Rayleigh-Taylor Instability", Phys. Fluids, 10, 297-307 (1967).

[8] F. H. Harlow and J. P. Shannon, "The Splash of a Liquid Drop", J. Comp. Phys. , 38, 3855-3866 (1967).

[9] B. J. Daly, "A Technique for Including Surface Tension Effects in Hydrodynamic Calculations", J. Comp. Phys. , 4, 97-117 (1969).

[10] J. R. Richards, A. M. Lenhoff and A. N. Beris, "Dynamics Breakup of liquidliquid jets", Phys. Fluids A, 6, 2640-2655 (1994).

[11] B. Lafaurie, C. Nardone, R. Scardovelli, S. Zaleski and G. Zanetti "Modeling Merging and Fragmentation in Multiphase Flows with SURFER", J. Comp. Phys. , 113, 134-147 (1994).

[12] M. J. Fritts and J. P. Boris, "The Lagrangian Solution of Transient Problems in Hydrodynamics Using a Triangular mesh", J. Comp. Phys. , 31, 173-215 (1979).

[13] D. E. Fyfe, E. S. Oran and M. J. Fritts, "Surface Tension and Viscosity with Lagrangian Hydrodynamics on a Triangular Mesh", J. Comp. Phys. , 76, 349384 (1988). 
[14] T. C. Jue and B. Ramaswamy "Cavity Natural Convection with a Deformable Free Surface", in Advances in Finite Element Analysis in Fluid Dynamics, (eds M. N. Dhaubhadel, et al.), ASME (1992).

[15] T. E. Tezduyar, M. Behr and J. Liou, "A New Strategy for Finite Element Computations Involving Moving Boundaries and Interfaces - The DeformingSpatial-Domain/Space-Time Procedure: I. The Concept and the Preliminary Numerical Tests", Comp. Meth. Appl. Mech. Engng. , 94, 339-351 (1992).

[16] T. E. Tezduyar, M. Behr, S. Mittal and J. Liou, "A New Strategy for Finite Element Computations Involving Moving Boundaries and Interfaces - The Deforming-Spatial-Domain/Space-Time Procedure: II. Computation of Freesurface Flows, Two-liquid flows, and flows with drifting cylinders", Comp. Meth. Appl. Mech. Engng., 94, 353-371 (1992).

[17] S. O. Unverdi and G. Tryggvason, "A Front-Tracking Method for Viscous, Incompressible, Multi-fluid Flows", J. Comp. Phys. , 100, 25-37 (1992).

[18] M. R. Nobari and G. Tryggvason, "Numerical Simulation of Drop Collisions", NASA TM 106751, ICOMP-94-23.

[19] M. R. Nobari and G. Tryggvason, "The Flow Induced by the Coalescence of Two Initially Stationary Drops", NASA TM 106752, ICOMP-94-24.

[20] B. N. Jiang, T. L. Lin and L. A. Povinelli, "Large-Scale Computation of Incompressible Viscous Flow by Least-Squares Finite Element Method", Comp. Meth. Appl. Mech. Engng., Vol 114, 213-231 (1994).

[21] B. N. Jiang, "Non-oscillatory and Non-diffusive Solution of Convection Problems by the Iterative Reweighted Least-Squares Finite Element Method" $J$. Comp. Phys. , 105, 108-121 (1993).

[22] S. T. Yu, B. N. Jiang, J. Wu and D. Jacqmin, "A Unified Approach for Simulation Free-Surface Flows by the Least-Squares Finite Element Method", Proceedings of the Sixth International Symposium on Computational Fluid Dynamics Sept. 1995, Nevada, USA.

[23] B. N. Jiang, C. Y. Loh and L. A. Povinelli, "Theoretical Study of the Incompressible Euler Equations by the Least-Squares Method", NASA TM 106535, ICOMP-94-24.

[24] L. Q. Tang and T. T. H. Tsang, "A Least-Squares Finite Element Method for Time-Dependent Incompressible Flows with Thermal Convection", Int. J. Num. Meth. Fluids, 17, 271-289 (1993). 
[25] G. F. Carey and B. N. Jiang, "Element-by-element Linear and Non-linear Solution Methods", Comm. Appl. Num. Meth., 2, 145-153 (1986).

[26] O. C. Zienkiewicz and J. Z. Zhu, "The Superconvergent Patch Recovery and A Posteriori Error Estimates. Part 1: The Recovery Technique", Int. J. Num. Meth. Engng. , 33, 1331-1364 (1992).

[27] O. C. Zienkiewicz and J. Z. Zhu, "A Simple Error Estimator and Adaptive Procedure for Practical Engineering Analysis", Int. J. Num. Meth. Engng., 24, 337-357 (1987).

[28] J. C. Martin and W. J. Moyce, "An Experimental Study of the Collapse of Liquid Columns on a Horizontal Plane", Philos. Trans. R. Soc. Lond. A, 244, 312-324 (1952).

[29] T. Nakayama and M. Mori, "An Eulerian Finite Element Method for TimeDependent Free Surface Problems in Hydrodynamics", Int. J. Num. Meth. Fluids , 22, 175-194 (1996).

[30] H. Haj-hariri, Q. Shi and A. Borhan, "Effect of Local Property Smearing on Global Variables: Implication for Numerical Simulations of Multiphase Flows", Phys. Fluids, 6, 2555-2257 (1994). 


\section{A The Governing Equations in Cylindrical Co- ordinate System}

The Navier-Stokes equations given by Eqs. (8)-(9) can be written in a cylindrical coordinate system as:

$$
\begin{aligned}
& \frac{\partial u_{r}}{\partial r}+\frac{1}{r} u_{r}+\frac{1}{r} \frac{\partial u_{\theta}}{\partial \theta}+\frac{\partial u_{z}}{\partial z}=0 \\
& \rho \frac{\partial u_{r}}{\partial t}-\rho \frac{u_{\theta}^{2}}{r}+\rho u_{r} \frac{\partial u_{r}}{\partial r}+\rho u_{\theta} \frac{1}{r} \frac{\partial u_{r}}{\partial \theta}+\rho u_{z} \frac{\partial u_{r}}{\partial z}+\frac{\partial p}{\partial r}+\mu\left(\frac{1}{r} \frac{\partial \omega_{z}}{\partial \theta}-\frac{\partial \omega_{\theta}}{\partial z}\right) \\
- & \left(2 \frac{\partial \mu}{\partial r} \frac{\partial u_{r}}{\partial r}+\frac{1}{r} \frac{\partial \mu}{\partial \theta}\left(\frac{1}{r} \frac{\partial u_{r}}{\partial \theta}+\frac{\partial u_{\theta}}{\partial r}-\frac{u_{\theta}}{r}\right)+\frac{\partial \mu}{\partial z}\left(\frac{\partial u_{r}}{\partial z}+\frac{\partial u_{z}}{\partial r}\right)\right)=f_{r} \\
& \rho \frac{\partial u_{\theta}}{\partial t}+\rho \frac{u_{r} u_{\theta}}{r}+\rho u_{r} \frac{\partial u_{\theta}}{\partial r}+\rho u_{\theta} \frac{1}{r} \frac{\partial u_{\theta}}{\partial \theta}+\rho u_{z} \frac{\partial u_{\theta}}{\partial z}+\frac{1}{r} \frac{\partial p}{\partial \theta}+\mu\left(\frac{\partial \omega_{r}}{\partial z}-\frac{\partial \omega_{z}}{\partial r}\right) \\
- & \left(\frac{\partial \mu}{\partial r}\left(\frac{1}{r} \frac{\partial u_{r}}{\partial \theta}+\frac{\partial u_{\theta}}{\partial r}-\frac{u_{\theta}}{r}\right)+2 \frac{1}{r} \frac{\partial \mu}{\partial \theta}\left(\frac{1}{r} \frac{\partial u_{\theta}}{\partial \theta}+\frac{u_{r}}{r}\right)+\frac{\partial \mu}{\partial z}\left(\frac{\partial u_{\theta}}{\partial z}+\frac{1}{r} \frac{\partial u_{z}}{\partial \theta}\right)\right) \\
& =f_{\theta} \\
& \rho \frac{\partial u_{z}}{\partial t}+\rho u_{r} \frac{\partial u_{z}}{\partial r}+\rho u_{\theta} \frac{1}{r} \frac{\partial u_{z}}{\partial \theta}+\rho u_{z} \frac{\partial u_{z}}{\partial z}+\frac{\partial p}{\partial z}+\mu\left(\frac{\partial \omega_{\theta}}{\partial r}+\frac{1}{r} \omega_{\theta}-\frac{1}{r} \frac{\partial \omega_{r}}{\partial \theta}\right) \\
- & \left(\frac{\partial \mu}{\partial r}\left(\frac{\partial u_{r}}{\partial z}+\frac{\partial u_{z}}{\partial r}\right)+\frac{1}{r} \frac{\partial \mu}{\partial \theta}\left(\frac{\partial u_{\theta}}{\partial z}+\frac{1}{r} \frac{\partial u_{z}}{\partial \theta}\right)+2 \frac{\partial \mu}{\partial z} \frac{\partial u_{z}}{\partial z}\right)=f_{z}
\end{aligned}
$$

The definition of $\boldsymbol{\omega}, \mathrm{Eq}$. (10), is given as:

$$
\begin{aligned}
& \omega_{r}=\frac{1}{r} \frac{\partial u_{z}}{\partial \theta}-\frac{\partial u_{\theta}}{\partial z} \\
& \omega_{\theta}=\frac{\partial u_{r}}{\partial z}-\frac{\partial u_{z}}{\partial r} \\
& \omega_{z}=\frac{\partial u_{\theta}}{\partial r}+\frac{1}{r} u_{\theta}-\frac{1}{r} \frac{\partial u_{r}}{\partial \theta}
\end{aligned}
$$

The compatibility condition, Eq. (11), in this system becomes:

$$
\frac{\partial \omega_{r}}{\partial r}+\frac{1}{r} \omega_{r}+\frac{1}{r} \frac{\partial \omega_{\theta}}{\partial \theta}+\frac{\partial \omega_{z}}{\partial z}=0
$$

The stress components due to surface tension are:

$$
\tau_{r \tau}=-\frac{\sigma}{[C]}\left(\frac{1}{|\nabla C|} \frac{\partial C}{\partial r} \frac{\partial C}{\partial r}-|\nabla C|\right)
$$




$$
\begin{aligned}
& \tau_{\theta \theta}=-\frac{\sigma}{[C]}\left(\frac{1}{|\nabla C|} \frac{1}{r^{2}} \frac{\partial C}{\partial \theta} \frac{\partial C}{\partial \theta}-|\nabla C|\right) \\
& \tau_{z z}=-\frac{\sigma}{[C]}\left(\frac{1}{|\nabla C|} \frac{\partial C}{\partial z} \frac{\partial C}{\partial z}-|\nabla C|\right) \\
& \tau_{r \theta}=\tau_{\theta r}=-\frac{\sigma}{[C]}\left(\frac{1}{|\nabla C|} \frac{1}{r} \frac{\partial C}{\partial r} \frac{\partial C}{\partial \theta}\right) \\
& \tau_{\theta z}=\tau_{z \theta}=-\frac{\sigma}{[C]}\left(\frac{1}{|\nabla C|} \frac{1}{r} \frac{\partial C}{\partial \theta} \frac{\partial C}{\partial z}\right) \\
& \tau_{r z}=\tau_{z r}=-\frac{\sigma}{[C]}\left(\frac{1}{|\nabla C|} \frac{\partial C}{\partial r} \frac{\partial C}{\partial z}\right)
\end{aligned}
$$

in which $|\nabla C|=\sqrt{\left(\frac{\partial u_{r}}{\partial r}\right)^{2}+\left(\frac{1}{r} \frac{\partial u_{r}}{\partial \theta}\right)^{2}+\left(\frac{\partial u_{x}}{\partial z}\right)^{2}}$.

The body force components due to the above stress tensor is:

$$
\begin{aligned}
& f_{r}=\frac{\partial \tau_{r r}}{\partial r}+\frac{1}{r}\left(\tau_{r r}-\tau_{\theta \theta}\right)+\frac{1}{r} \frac{\partial \tau_{r \theta}}{\partial \theta}+\frac{\partial \tau_{r z}}{\partial z} \\
& f_{\theta}=\frac{\partial \tau_{r \theta}}{\partial r}+\frac{1}{r} \frac{\partial \tau_{\theta \theta}}{\partial \theta}+\frac{2}{r} \tau_{r \theta}+\frac{\partial \tau_{\theta z}}{\partial z} \\
& f_{z}=\frac{\partial \tau_{r z}}{\partial r}+\frac{1}{r} \frac{\partial \tau_{\theta z}}{\partial \theta}+\frac{1}{r} \tau_{r z}+\frac{\partial \tau_{z z}}{\partial z}
\end{aligned}
$$

When the flow is axisymmetric all the $\theta$-derivatives become zero. Further if the flow is non-swirling, $u_{\theta}$ becomes zero. For such cases the governing equations are further simplified. 
Public reporting burden for this collection of information is estimated to average 1 hour per response. including the time for reviewing instructions, searching existing dala sources,

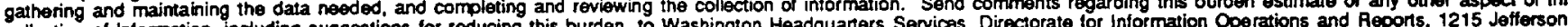

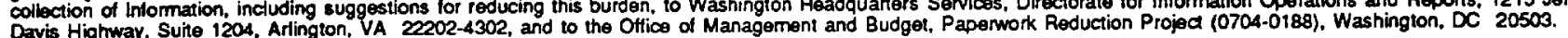

\begin{tabular}{|l|l|l|}
\hline 1. AGENCY USE ONLY (Leave blank) & $\begin{array}{r}\text { 2. REPORT DATE } \\
\text { December } 1996\end{array}$ & $\begin{array}{r}\text { 3. REPORT TYPE AND DATES COVERED } \\
\text { Contractor Report }\end{array}$
\end{tabular}

\section{TITLE AND SUBTITLE}

Simulation of Two-Fluid Flows by the Least-Squares Finite Element Method Using a Continuum Surface Tension Model

6. AUTHOR(S)

Jie Wu, Sheng-Tao, and Bo-nan Jiang

\section{PERFORMNG ORGANIZATION NAME(S) AND ADDRESS(ES)}

Institute for Computational Mechanics in Propulsion

22800 Cedar Point Road

Cleveland, Ohio 44142

9. SPONSORINGMONITORING AGENCY NAME(S) AND ADDRESS(ES)

National Aeronautics and Space Administration

Lewis Research Center

Cleveland, Ohio 44135-3191
5. FUNDING NUMBERS

WU-505-90-5K

NCC3-483

\section{SUPPLEMENTARY NOTES}

Jie Wu and Bo-nan Jiang, Institute for Computational Mechanics in Propulsion, NASA Lewis Research Center (work performed under NASA Cooperative Agreement NCC3-483); Sheng-Tao, NYMA, Inc., 2001 Aerospace Parkway, Brook Park, Ohio 44142 (work performed under NASA Contract NAS3-27186). ICOMP Program Director, Louis A. Povinelli, organization code 2600, (216) 433-5818.

12a. DISTRIBUTIONAVAILABILITY STATEMENT 12b. DISTRIBUTION CODE

Unclassified - Unlimited

Subject Category 64

This publication is available from the NASA Center for AeroSpace Information, (301) 621-0390.

13. ABSTRACT (Naximum 200 words)

In this paper a numerical procedure for simulating two-fluid flows is presented. This procedure is based on the Volume of Fluid (VOF) method proposed by Hirt and Nichols and the continuum surface force (CSF) model developed by Brackbill, et al. In the VOF method fluids of different properties are identified through the use of a continuous field variable (color function). The color function assigns a unique constant (color) to each fluid. The interfaces between different fluids are distinct due to sharp gradients of the color function. The evolution of the interfaces is captured by solving the convective equation of the color function. The CSF model is used as a means to treat surface tension effect at the interfaces. Here a modified version of the CSF model, proposed by Jacqmin, is used to calculate the tension force. In the modified version, the force term is obtained by calculating the divergence of a stress tensor defined by the gradient of the color function. In its analytical form, this stress formulation is equivalent to the original CSF model. Numerically, however, the use of the stress formulation has some advantages over the original CSF model, as it bypasses the difficulty in approximating the curvatures of the interfaces. The least-squares finite element method (LSFEM) is used to discretize the governing equation systems. The LSFEM has proven to be effective in solving incompressible Navier-Stokes equations and pure convection equations, making it an ideal candidate for the present applications. The LSFEM handles all the equations in a unified manner without any additional special treatment such as upwinding or artificial dissipation. Various bench mark tests have been carried out for both two dimensional planar and axisymmetric flows, including a dam breaking, oscillating and stationary bubbles and a conical liquid sheet in a pressure swirl atomizer.

14. SUBJECT TERMS

Least-squares; Finite element method; Two-fluid flow 15. NUMEER OF PAGES 26 16. PRICE CODE A03

\begin{tabular}{|c|c|}
\hline $\begin{array}{c}\text { 17. SECURTY CLASSIFICATION } \\
\text { OF REPORT } \\
\text { Unclassified }\end{array}$ & $\begin{array}{c}\text { 18. SECURTY CLASSIFICATION } \\
\text { OF THIS PAGE } \\
\text { Unclassified }\end{array}$ \\
\hline
\end{tabular}

19. SECURTYY CLASSIFICATION OF ABSTRACT Unclassified 
